\title{
Lenalidomide - A Brief Review of its Use in Myelodysplastic Syndromes
}

\author{
Jaykumar J Sejpal, ${ }^{1}$ Yogesh R Belagali² and Hanmant V Barkate $^{3}$ \\ 1. Senior Medical Advisor (Researcher); 2. Medical Advisor (Researcher); 3. Vice President (Researcher), Department of Medical Affairs \& Clinical Research, \\ Intas Pharmaceuticals Limited, Ahmedabad, India
}

\begin{abstract}
Myelodysplastic syndromes (MDS) are a heterogeneous group of blood diseases mainly affecting older people. Deletion of the long arm of chromosome 5 (del[5q]) is reported in approximately 10-15\% of all MDS cases. Lenalidomide is an immunomodulatory, anti-cytokine and anti-angiogenic agent, which leads to red blood cells transfusion independence in patients of lower risk MDS with del(5q). This review briefly describes role of lenalidomide in treatment of lower-risk MDS with del(5q) as well as non-del-5q MDS. Recent evidence also suggest a potential role of lenalidomide in combination with other agents for treatment of higher-risk MDS.
\end{abstract}

\section{Keywords}

Lenalidomide, myelodysplastic syndrome, 5q deletion, MDS-003 trial, MDS-004 trial, lenalidomide combination with azacitidine

Disclosure: Jaykumar J Sejpal, Yogesh R Belagali and Hanmant V Barkate are employees of Intas Pharmaceuticals Limited. No funding was received in support of the publication of this article.

Open Access: This article is published under the Creative Commons Attribution Noncommercial License, which permits any non-commercial use, distribution, adaptation and reproduction provided the original author(s) and source are given appropriate credit.

Received: 31 August 2015 Accepted: 26 October 2015 Citation: European Oncology \& Haematology, 2015;11(2):141-6 DOI: http://doi.org/10.17925/EOH.2015.11.02.141 Correspondence: Jaykumar J Sejpal, Intas Pharmaceuticals Limited, Ahmedabad, 380054, India. E: jay_sejpal2006@yahoo.co.in

\section{Myelodysplastic Syndromes and 5q Deletion Myelodysplastic syndromes (MDS) are a group of rare blood disorders that occur as a result of abnormal development of blood cells within the bone marrow. MDS may progress to life-threatening failure of the bone marrow or develop into an acute leukaemia. It affects, most commonly, the elderly population, with median age at diagnosis of about 65 years. MDS are a heterogeneous group of malignant haematopoietic stem cell disorders, which are characterised by dysplasia and ineffective production of blood cells, and increased risk of transformation to acute myeloid leukaemia (AML). Patients with MDS have variable cytopenias, i.e. reduction in the production of normal red blood cells (RBCS), platelets and white blood cells (mainly neutrophils). This may present as anaemia, bleeding and an increased risk of infection. ${ }^{1,2}$}

The incidence of MDS in the US is estimated to be about 4.1/100,000 inhabitants/year. The incidence rises with increasing age: 4.9 for people aged 50 to 70 years; 22.8 for people older than 70 years. ${ }^{3}$ The incidence of MDS in Europe is approximately $4 / 100,000$ inhabitants/year (increasing to $40-50 / 100,000$ in patients aged $\geq 70$ years). While there are no ethnic differences in the incidence of MDS, Asians tend to have MDS at an earlier age, more often show a hypo cellular marrow and less commonly present with isolated $5 q$ deletion ( $5 q$ - syndrome), while more commonly present with trisomy 8 compared with Western populations. ${ }^{4}$

The International Prognostic Scoring System (IPSS), which is the most commonly used tool for MDS, defines four prognostic risk categories: good, intermediate-1, intermediate-2 and poor, based on the number of cytopenias, proportion of marrow blasts and cytogenetic abnormalities. ${ }^{5}$ The revised version of IPSS defines five prognostic risk categories: very low, low, intermediate, high and very high, by further refining the same prognostic variables. ${ }^{6}$

Cytogenetic abnormalities can be detected in bone marrow cells in approximately $30-50 \%$ of MDS patients. The most common chromosomal abnormalities seen in MDS are deletion of the long arm of chromosome 5 (del[5q]), -7 or del(7q), trisomy 8, del(20q) and loss of the Y chromosome.? del(5q) has been reported to be present in approximately $10-15 \%$ of all MDS cases either as the sole karyotype abnormality or as part of a more complex karyotype and it has distinct clinical implications for MDS. ${ }^{8}$

'MDS associated with isolated del( $5 q)$ ' is recognised by The World Health Organisation (WHO) as a distinct entity, ${ }^{9}$ and it is characterised by an isolated del(5q), female predominance, macrocytic anaemia, $<5 \%$ bone marrow blasts, $<1 \%$ of peripheral blood blasts, a normal or increased platelet count and hypolobated megakaryocytes and no Auer rods. Patients with isolated del(5q) MDS have a stable clinical course and rare progression to AML. It has a relatively good prognosis and good chance of responding to treatment with lenalidomide. ${ }^{10}$ In comparison, del(5q) or structural rearrangements of $5 q$ with other chromosomal changes lead to higher-risk MDS and worse outcomes. ${ }^{11}$

\section{Profile of Lenalidomide}

Lenalidomide, a thalidomide analogue, is an orally administered immunomodulator with anti-angiogenic, antineoplastic, anti-inflammatory and pro-erythropoietic properties. ${ }^{12}$ Lenalidomide was developed in effort to avoid thalidomide side effects, and to increase efficacy. Lenalidomide shares a number of structural and biological properties with thalidomide but it is safer and more potent than thalidomide. ${ }^{13}$ 
Table 1: Key Patient Characteristics, Criteria for Inclusion and Exclusion and Study Methodologies of MDS-003 and MDS-004 Trials

$\begin{array}{lll}\text { Parameter } & \text { MDS-003 }(\mathrm{n}=148) & \text { MDS-004 (n=205) } \\ \begin{array}{l}\text { Patient characteristics } \\ \text { Median age in years (range) }\end{array} & 71(37-95) & 69(36-86) \\ \quad \text { RBC transfusion burden in previous } & 6 & 6 \\ 8 \text { weeks (units) } & & 48.9 \% \\ \text { IPSS risk category (\% patients) } & 37.0 \% & 51.1 \% \\ \quad \text { Low } & 44.0 \% & 76.3 \% \\ \quad \text { Intermediate-1 } & & 23.7 \%\end{array}$

\section{Key inclusion criteria}

del $(5 q) \pm \geq 1$ cytogenetic abnormality; IPSS low- or intermediate-1-risk; anaemia requiring RBC transfusions ( $\geq 2$ units in the 8 weeks prior to enrolment ${ }^{21}$ or no period of 8 consecutive weeks without RBC transfusions within the 16 weeks prior to randomisation 22

\section{Key exclusion criteria}

Proliferative chronic myelomonocytic leukaemia $(12,000$ leukocytes $/ \mu \mathrm{L})$; severe neutropenia ( $<500$ neutrophils/ $\mu \mathrm{L})$; severe thrombocytopenia $\left(<25,000^{21}\right.$ or $<50,000^{22}$ platelets/ $\left.\mu \mathrm{L}\right)$

\section{Study methods}

Treatment

Treatment Duration
- Patients received $10 \mathrm{mg}$ of lenalidomide for 21 days every 4 weeks or daily

- Lenalidomide was given for 24 weeks in trial and responders continued to receive treatment until treatment failure, progression or unacceptable tolerability

- Eligible patients underwent stratification according to IPSS score and were then randomised to lenalidomide $10 \mathrm{mg} /$ day on days 1-21 ( $n=69)$, lenalidomide $5 \mathrm{mg} /$ day on days 1-28 $(n=69)$ or placebo on days $1-28$ of 28 -day cycles $(n=67)$

- Crossover to lenalidomide or higher dose was allowed for patient who did not have at least a minor erythroid response by week 16

- Patients who completed 2 weeks of double-blind treatment without disease progression or erythroid relapse were eligible to receive open-label treatment with lenalidomide at their current dosage for up to 156 weeks

\section{Study endpoints}

Primary endpoint

Secondary endpoints
Proportion of patients achieving RBC-TI for $\geq 8$ consecutive weeks with an associated increase in haemoglobin concentration of $\geq 1 \mathrm{~g} / \mathrm{dL}$ Duration of transfusion independence; the frequency of minor erythroid, cytogenetic and pathological responses
Proportion of patients achieving RBC-TI for $\geq 26$ consecutive weeks

Erythroid response, duration of RBC-TI, cytogenetic response, OS, AML progression, safety and HRQOL

$A M L=$ acute myeloid leukaemia; Del(5q) = deletion of the long arm of chromosome 5; HRQoL = health-related quality of life; IPSS = International Prognostic Scoring System; MDS = myelodysplastic syndromes; $O S=$ overall survival; $R B C=$ red blood cell; $T I=$ transfusion independence .

The key therapeutic mechanisms of action of lenalidomide include stimulation of erythropoiesis, immunomodulatory effects and antineoplastic effects, such as anti-angiogenic and antiproliferative activity. Immunomodulatory effects of lenalidomide mainly include: increasing the number and activity of T-cells, natural killer (NK) and NK T-cells; inhibition of the pro-inflammatory cytokines, such as tumour necrosis factor (TNF)- $\alpha$; and, increasing the production of the anti-inflammatory cytokine interleukin (IL)-10 in peripheral blood mononuclear cells. ${ }^{14,15}$

In del(5q) MDS, lenalidomide causes a cytotoxic effect by stabilisation of the mouse double minute 2 homolog (MDM2) gene, and thereby leading to accelerated p53 degradation. ${ }^{16}$ In MDS with non-del(5q), lenalidomide acts by modifying bone marrow microenvironment, which abolishes the function of pro-apoptotic and pro-inflammatory cytokines an increased expression of adhesion and restoring erythropoiesis via erythropoietin (EPO)-induced activation of signal transducer and activator of transcription (STAT5) gene. ${ }^{17,18}$
Lenalidomide inhibits angiogenesis by inhibiting the migration and adhesion of endothelial cells induced by cytokines, such as vascular endothelial growth factor and basic fibroblast growth factor. In vitro, lenalidomide inhibits the expression of cyclooxygenase (COX)-2, but

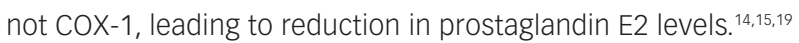

\section{Therapeutic Efficacy of Lenalidomide in Myelodysplastic Syndromes}

Lenalidomide was first studied in a single-centre 16-week, phase I/II trial. ${ }^{20}$ This study included 43 patients with MDS and who had transfusion-dependence or symptomatic anaemia. All patients were refractory to EPO or had high endogenous EPO levels. Lenalidomide was administered in three different dosing schedules: $25 \mathrm{mg}$ daily, 10 $\mathrm{mg}$ daily and $10 \mathrm{mg} /$ day for 21 days of a 28-day cycle. Results of this study showed the potential of lenalidomide in EPO-resistant del(5q) MDS as well as the role of lenalidomide in EPO-refractory non-del( $5 q)$ MDS patients. Eighty-three per cent of patients with del(5q) MDS showed major erythroid responses, defined as sustained transfusion 
independence, compared with $53 \%$ of patients with normal karyotype and $12 \%$ of patients with other karyotype abnormalities. Dosedependent myelosuppression (neutropenia, thrombocytopenia) was the most common adverse event (AE); well-tolerated dose was $10 \mathrm{mg}$ daily for 21 days of each 28-day cycle.

\section{Lenalidomide in Myelodysplastic Syndromes Associated with del(5q) - Landmark Trials}

Based on results of study mentioned above, the clinical efficacy of lenalidomide in patients with MDS associated with del(5q) was evaluated in two registration trials: the non-comparative, phase II, MDS-003 trial ( $n=148$; US registration trial) ${ }^{21}$ and the phase III, double-blind, placebo-controlled, multicentre, MDS-004 trial $(n=205)$ conducted in Europe and Israel. ${ }^{22}$

These landmark trials21,22 included adult patients with IPSS low- or intermediate-1-risk (lower risk) MDS associated with del(5q), with or without additional cytogenetic abnormalities. Included patients had RBC transfusion-dependent (TI) anaemia. Key patient characteristics, criteria for inclusion and exclusion and study methodologies are given in Table 1.

\section{Results}

\section{Erythroid Response}

In the MDS-004 trial, ${ }^{22}$ a significantly higher proportion of patients in lenalidomide 10 and $5 \mathrm{mg}$ groups experienced RBC-TI for $>26$ weeks versus placebo (56.1\% and $42.6 \%$, respectively, versus $5.9 \%$; both $\mathrm{p}<0.001$ ) (see Figure 1). While in the MDS-003 trial, $67 \%$ of patients receiving lenalidomide $10 \mathrm{mg} /$ day achieved $\mathrm{RBC}$-TI for $\geq 8$ consecutive weeks and an associated $\geq 1 \mathrm{~g} / \mathrm{dL}$ increase in haemoglobin level (see Figure 2). ${ }^{21}$

\section{Subgroup Analysis of Erythroid Response}

In MDS-004, ${ }^{22}$ subgroup analysis suggested that erythroid response (RBC-TI for $\geq 26$ week) rates favoured lenalidomide $10 \mathrm{mg}$ over $5 \mathrm{mg}$ for most subgroups. Particularly, in patients with baseline EPO levels $>500 \mathrm{mIU} / \mathrm{mL}$, the RBC-TI rate was significantly higher with lenalidomide $10 \mathrm{mg}$ versus $5 \mathrm{mg}$ (76.2\% versus $33.3 \%$; $\mathrm{p}=0.004)$.

\section{Time to Achieve Erythroid Response}

Erythroid responses to lenalidomide treatment were achieved rapidly. In the MDS-004 trial, in patients who achieved RBC-TI for $\geq 26$ consecutive weeks with lenalidomide treatment, the onset of response occurred in $86 \%$ of responders during cycle one $(48.8 \%)$ or cycle two $(37.2 \%) .{ }^{22}$ This finding was consistent with that observed in the MDS-003 trial, in which the median time to an erythroid response was 4.6 weeks. ${ }^{21}$

\section{Increase in Haemoglobin}

In MDS-00321, with lenalidomide $10 \mathrm{mg} /$ day treatment, haemoglobin concentration increased from a median minimum of $7.8 \mathrm{~g} / \mathrm{dL}$ during the baseline period to a median maximum of $13.4 \mathrm{~g} / \mathrm{dL}$ during RBC-TI response period, with a corresponding median rise from baseline of $5.4 \mathrm{~g} / \mathrm{dL}$. In MDS-004, ${ }^{22}$ the median increase from baseline (value not reported) in maximum haemoglobin concentrations were 5.2 and $6.3 \mathrm{~g} /$ $\mathrm{dL}$ with lenalidomide 5 and $10 \mathrm{mg}$ regimens, respectively.

Duration of Red Blood Cell Transfusion Independence The median duration of RBC-TI for $\geq 8$ consecutive weeks was not reached in either of these trials, and in MDS-004, the median duration of RBC-TI for $\geq 26$ consecutive weeks was also not reached. ${ }^{22}$
Figure 1: Efficacy of Oral Lenalidomide in Patients with Chromosome $5 q$ Deletion-associated Myelodysplastic Syndrome

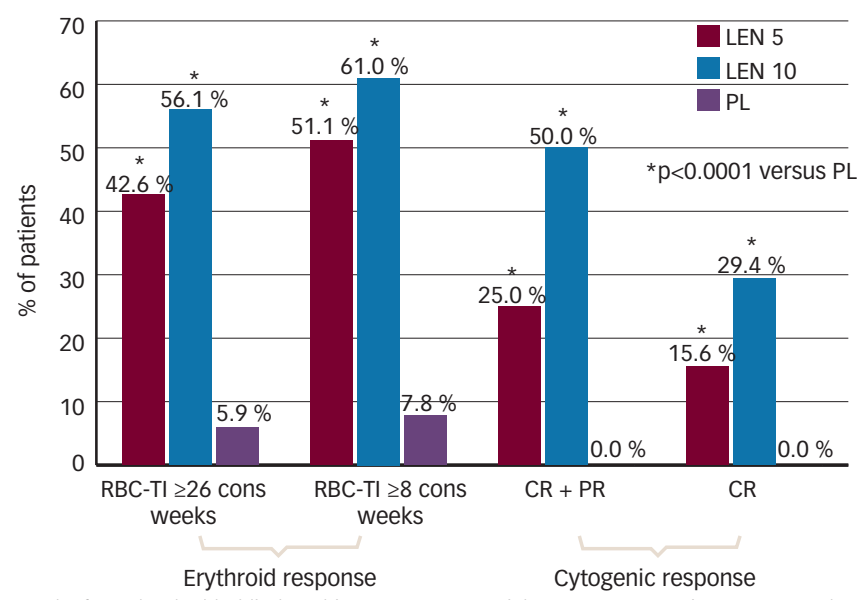

Results from the double-blind, multicentre MDS-004 trial. cons = consecutive; $C R=$ complet response; $L E N=$ lenalidomide; $P L=$ placebo; $P R=$ partial response; $R B C-T I=$ red blood celltransfusion independence.

Figure 2: Efficacy of Oral Lenalidomide in Patients with Chromosome $5 q$ Deletion-associated Myelodysplastic Syndrome

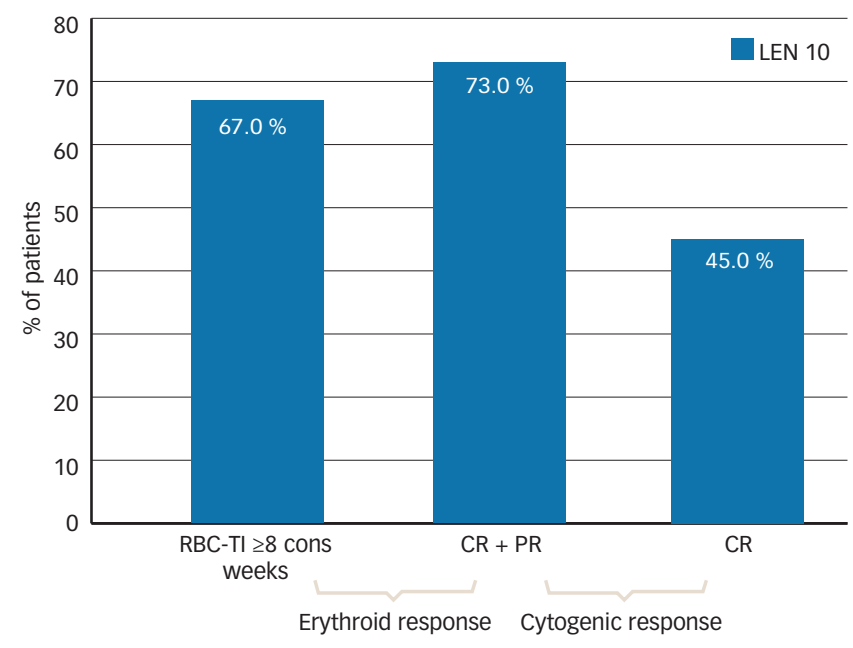

Results from non-comparative MDS-003 trial. cons = consecutive; $C R=$ complete response; $L E N=$ lenalidomide; $P L=$ placebo; $P R=$ partial response; $R B C-T I=$ red blood celltransfusion independence.

Also, 60-67\% responders remained transfusion free at median followup of 1.55 years.

\section{Cytogenetic Response}

In MDS-004, ${ }^{22}$ treatment with lenalidomide 5 or $10 \mathrm{mg}$ regimens resulted in significantly higher cytogenetic response rates than placebo (see Figure 1). With lenalidomide $10 \mathrm{mg}$ regimens, complete plus partial cytogenetic responses were achieved by $50 \%$ and $73 \%$ of patients and a complete cytogenetic remission was seen in $29 \%$ and $45 \%$ of patients in the MDS-004 22 and MDS-00321 trials, respectively (see Figures 1 and 2).

\section{Disease Progression and Overall Survival}

In the MDS-004 trial, ${ }^{22}$ after median follow-up of 30.9-36.1 months (double-blind and open-label treatment periods), the proportion of patients progressed to AML during the were $23.2 \%$ and $21.7 \%$ of patients receiving lenalidomide 5 or $10 \mathrm{mg}$ regimens, $30.4 \%$ of those randomised to placebo who crossed over to lenalidomide $5 \mathrm{mg} /$ day 
Table 2: Tolerability of Oral Lenalidomide in Patients with Myelodysplastic Syndrome with $5 q$ Deletion

\begin{tabular}{|c|c|c|c|c|}
\hline \multirow[t]{3}{*}{ Incidence (\%) } & \multicolumn{3}{|l|}{ MDS-004 } & \multirow{2}{*}{$\begin{array}{l}\text { MDS-003 } \\
\text { Lenalidomide } \\
10 \mathrm{mg}\end{array}$} \\
\hline & $\begin{array}{l}\text { Lenalidomide } \\
5 \mathrm{mg}\end{array}$ & $\begin{array}{l}\text { Lenalidomide } \\
10 \mathrm{mg}\end{array}$ & Placebo & \\
\hline & $\mathrm{n}=69$ & $\mathrm{n}=69$ & $n=67$ & $\mathrm{n}=148$ \\
\hline \multicolumn{5}{|c|}{ Common Grade 3 or 4 AEs (incidence $\geq 5 \%$ ) } \\
\hline Neutropenia & $73.9 \%$ & $75.4 \%$ & $14.9 \%$ & $55.0 \%$ \\
\hline Thrombocytopenia & $33.3 \%$ & $40.6 \%$ & $1.5 \%$ & $44.0 \%$ \\
\hline Leukopenia & $13.0 \%$ & $8.7 \%$ & $0.0 \%$ & $6.0 \%$ \\
\hline Anaemia & $5.8 \%$ & $2.9 \%$ & $9.0 \%$ & $7.0 \%$ \\
\hline $\begin{array}{l}\text { Deep-vein } \\
\text { thrombosis }\end{array}$ & $1.4 \%$ & $5.8 \%$ & $1.5 \%$ & $3.0 \%$ \\
\hline Rash & - & - & - & $6.0 \%$ \\
\hline Infection & $9.0 \%$ & $16.0 \%$ & - & - \\
\hline $\begin{array}{l}\text { Dosage reduction } \\
\text { due to AES }\end{array}$ & $52.2 \%$ & $55.1 \%$ & $0.0 \%$ & $\begin{array}{l}84.0 \% \text { (91.0\% } \\
\text { in daily dose, } \\
67.0 \% \text { in 21-day } \\
\text { dose) }\end{array}$ \\
\hline Discontinuation & $17.4 \%$ & $8.7 \%$ & $4.5 \%$ & $20.0 \%$ \\
\hline
\end{tabular}

AES $=$ adverse events; $M D S=$ myelodysplastic syndromes.

and $36.4 \%$ of those receiving placebo throughout the study. Overall risk of AML progression for the lenalidomide dose groups combined was $16.8 \%$ (95\% confidence interval [Cl], 9.8-23.7) at 2 years and $25.1 \%$ (95\% Cl 17.1-33.1) at 3 years.

At a median follow-up of 35.5-36.9 months, median overall survival (OS) duration was $\geq 35.5$ (95\% Cl 24.6 - not reached), 44.5 (95\% Cl 35.5 - not reached) and 42.4 (95\% Cl 31.9 - not reached) months with lenalidomide 5 and $10 \mathrm{mg}$ regimens and placebo, respectively. ${ }^{22}$

A retrospective analysis by Kuendgen et al., ${ }^{23}$ compared long-term outcomes in lenalidomide-treated and untreated patients with MDS with del(5q). This analysis used data of 295 lenalidomide-treated patients from two clinical trials (MDS-003 and MDS-004) and 125 untreated and RBC-transfusion-dependent del(5q) MDS patients (IPSS low- or Intermediate-1-risk category) from a large multicentre registry. The 2-year cumulative AML progression rates $(95 \% \mathrm{Cl}$ ) in the lenalidomide-treated versus untreated cohorts were $6.9 \%$ (93.3-13.9) and $12.1 \%$ (7.0-20.3). Two-year OS probabilities (95\% Cl) were $89.9 \%$ (84.1-96.0) and $74.4 \%$ (66.1-83.7), respectively. AML progression risk did not significantly differ in both groups (hazard ratio [HR] 0.969, $\mathrm{p}=0.930$ ); however, treatment with lenalidomide led to significant

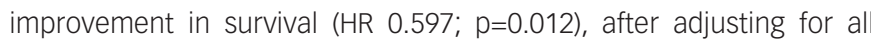
other covariates. Thus, lenalidomide treatment did not increase the risk of $\mathrm{AML}$ progression and conferred a possible survival benefit in RBC transfusion-dependent patients with lower risk del(5q) MDS.

\section{Health-related Quality of Life}

Health-related quality of life (HRQOL) was assessed in the MDS-004 trial, using the patient-reported Functional Assessment of Cancer TherapyAnemia (FACT-An) questionnaire. After 12 weeks of treatment (before non-responders were crossed over to lenalidomide) mean change of FACT-An total scores from baseline was significantly improved following treatment with lenalidomide 5 and $10 \mathrm{mg}$ ( +5.7 and +5.7 , respectively) versus placebo $(-2.8)$ (both $\mathrm{p}<0.05)$. Clinically important changes in
HRQOL from baseline were observed at weeks 12, 24, 36 and 48 among $\mathrm{RBC}-\mathrm{TI} \geq 26$ week responders in both lenalidomide groups. ${ }^{24,25}$

\section{Tolerability}

Oral lenalidomide (up to a dose of $10 \mathrm{mg} /$ day) has a manageable safety profile in patients with del(5q)- MDS (see Table 2). ${ }^{21,22}$ Although many patients on lenalidomide experienced at least one grade 3 or 4 AEs in clinical trials, most were manageable with a dosage reduction and only $\leq 20 \%$ of patients discontinued treatment because of AEs.

In the MDS-004 or MDS-003 trial, the most commonly reported (incidence $\geq 10 \%$ ) grade 3 or 4 AEs with lenalidomide treatment were neutropenia, thrombocytopenia, leukopenia and infection. Most common adverse reactions (>15\%) include thrombocytopenia, neutropenia, diarrhoea, pruritus, rash, fatigue, constipation, nausea, nasopharyngitis, arthralgia, pyrexia, back pain, peripheral oedema, cough, dizziness, headache, muscle cramp, dyspnoea, pharyngitis and epistaxis. Grade 3 or 4 neutropenia and thrombocytopenia occurred more frequently during the initial two cycles of lenalidomide treatment and the incidence decreased with further cycles.

\section{Lenalidomide in Non-del-5q Myelodysplastic Syndromes}

MDS-002 was a multicentre, phase ॥ trial evaluating lenalidomide therapy for transfusion-dependent patients with low- or int-1-risk (lower risk) MDS without any or with non-del(5q) chromosomal abnormalities $(\mathrm{n}=214){ }^{26}$ Patients received oral lenalidomide $10 \mathrm{mg}$ daily or $10 \mathrm{mg} /$ day for 21 of a 28 -day cycle. The most common grade 3/4 AEs were neutropenia (30\%) and thrombocytopenia (25\%).

According to an intention-to-treat analysis, $26 \%$ patients achieved TI after a median of 4.8 weeks of treatment. Median duration of TI was 41.0 weeks. In patients who achieved TI, the median rise in haemoglobin was $3.2 \mathrm{~g} / \mathrm{dL}$ (range, 1.0-9.8 $\mathrm{g} / \mathrm{dL}$ ) from baseline. Overall rate of haematological improvement $(\mathrm{HI})$ ( $\mathrm{TI}$ response $+\geq 50 \%$ reduction in transfusion requirement) was $43 \%$. Lenalidomide was found to have clinically meaningful activity in transfusion-dependent patients with lower-risk non-del-5q MDS.

MDS-005 was a multicentre, double-blind, parallel-group international phase III study, which compared lenalidomide with placebo in RBCtransfusion dependent lower-risk MDS without del(5q) patients, who were unresponsive or refractory to EPO-stimulating agents (ESA). ${ }^{27}$ Patients were randomised in 2:1 ratio; to oral lenalidomide, $10 \mathrm{mg}$ once daily $(n=160)$ versus placebo $(n=79)$. The primary endpoint was RBC-TI of $\geq 56$ days, which was defined as absence of any RBC transfusions during any 56 consecutive days. Secondary endpoints were time to TI, duration of $\mathrm{TI}, \mathrm{TI}$ of $\geq 168$ days, progression to AML, OS and safety. Significantly more patients on lenalidomide achieved RBC-TI $\geq 56$ days versus placebo (26.9\% versus $2.5 \%$; $p<0.001$ ). Ninety per cent of patients with TI responded within 16 weeks of treatment. For patients who became transfusion independent, the median duration of TI was 8.2 months (range 5.2-17.8). Additionally, RBC-TI $\geq 168$ days was achieved in $17.5 \%$ in the lenalidomide group and $0 \%$ of patients in placebo group. The incidence of AML progression (per 100 person-years) was 1.91 (95\% $\mathrm{Cl}$ 0.80-4.59) and 2.46 (95 \% Cl 0.79-7.64) for lenalidomide and placebo patients, respectively. The follow-up period was not long enough to analyse OS comparison. In the study, the most common AEs associated with lenalidomide were related to myelosuppression. Grade $3 / 4$ neutropenia occurred in $61.9 \%$ and $12.7 \%$ in the lenalidomide and 
placebo groups, respectively, and grade 3/4 thrombocytopenia occurred in $35.6 \%$ versus $3.8 \%$. This study provided new insights into the clinical results of lenalidomide in non-del-5q patients. Lenalidomide may offer this refractory patient population an additional option beyond their current limited choices.

\section{Combination Therapy in Higher Risk Myelodysplastic Syndromes}

With lenalidomide's success in lower-risk del(5q) MDS, it was further investigated for the treatment of higher-risk MDS. However, due to modest activity of lenalidomide monotherapy in higher-risk MDS, studies on lenalidomide in combination with other agents were required.

Thus, research was initiated with lenalidomide and azacitidine combination in MDS, since they work on different targets involved in the disease pathogenesis. In view of the single-agent activity of azacitidine and lenalidomide in MDS and AML, it was hypothesised that the combination of these two agents could potentially improve outcomes over monotherapy with either agent alone. Lenalidomide as immunomodulatory agent targets the microenvironment, while azacitidine works on DNA and RNA methylation. A multicentre phase II trial evaluated the use of combination azacitidine at $75 \mathrm{mg} / \mathrm{m}^{2}$ for 5 days with lenalidomide $10 \mathrm{mg} /$ day for 21 days of 28 -day cycle. ${ }^{28}$ This trial specifically targeted higher-risk MDS patients; total 36 patients: five patients IPSS intermediate-1, 20 patients IPSS intermediate-2 and 11 patients IPSS high. Overall response rate was $72 \%$, out of which $44 \%$ complete response (CR) and $28 \%$ with HI. Median OS was over 37 months for CR and 13.6 months for the entire study population. Commonly reported AEs were febrile neutropenia (22\%), infection (11\%), pulmonary AE (11\%), cardiac AE (11\%), constitutional (11\%) and dermatological AE (11\%). Thus, lenalidomide/azacitidine combination was found to be welltolerated and highly active in treatment of higher-risk MDS. ${ }^{28}$

A phase II trial by Sekeres et al. compared the addition of vorinostat or lenalidomide to azacitidine, with azacitidine alone. This study included 276 evaluable patients with higher-risk MDS, defined as intermediate-2 or high-risk IPSS score and/or bone marrow blasts $\geq 5 \%$ $(n=226)$, as well as patients with chronic myelomonocytic leukaemia (CMML) and $<20 \%$ blasts $(n=50) .{ }^{29}$ Patients were randomised to one of three treatment arms: azacitidine $75 \mathrm{mg} / \mathrm{m}^{2}$ on days $1-7$ of each 28-day cycle $(n=92)$; azacitidine plus lenalidomide $10 \mathrm{mg} /$ day on days 1-21 ( $n=93$ ); or azacitidine plus vorinostat $300 \mathrm{mg}$ twice daily (BID) on days 3-9 $(n=91)$. The overall response rates were similar for all patients regardless of treatment arm; single-agent azacitidine ( $36 \%$ ), azacitidine plus lenalidomide ( $37 \% ; p=1.0$ versus azacitidine monotherapy), or azacitidine plus vorinostat ( $22 \% ; p=0.07$ versus azacitidine monotherapy). Also, the proportion of patients achieving $C R$, partial response (PR) and $\mathrm{HI}$ were similar across all treatment groups. Thus, compared with azacitidine alone, the combination of vorinostat or lenalidomide with azacitidine failed to improve response in patients with higher-risk MDS and CMML.

The dose and schedule for azacitidine-lenalidomide combination used by sekeres et al. might not be optimum. ${ }^{29}$ As lenalidomide inhibits cell cycle progression and effectiveness of azacitidine is dependent on cycling cells, sequential administration of azacitidine followed by lenalidomide might increase efficacy. To study such a schedule, DiNardo et al. ${ }^{30}$ conducted a phase $1 / /$ trial of the sequential combination of azacitidine followed by lenalidomide in high-risk patients with MDS and AML. During phase I and IIa, patients with relapsed or refractory
AML or MDS with bone marrow blasts more than $10 \%$ were included. Phase IIb included previously untreated patients with higher-risk MDS with up to $30 \%$ blasts. All participants received $75 \mathrm{mg} / \mathrm{m}^{2}$ azacitidine once a day for days 1-5 for each 28-day cycle. Oral lenalidomide was given for 5 or 10 days starting on day 6 in seven different doses in phase I design $(n=28)$. In total 88 patients were enrolled, 28 in phase I and 60 in phase II. A maximum tolerated dose of lenalidomide in combination with azacitidine was initially considered as $50 \mathrm{mg}$ per day for 10 days. However, due to the high rate of myelosuppression and myelosuppression-related toxic effects in the first 20 patients in phase II, the lenalidomide dose was revised to $25 \mathrm{mg}$ per day for 5 days. Also in phase II patients with less than $30 \%$ blasts were included to focus mainly on MDS. Median OS was 75 weeks for the 40 patients in phase IIb. The most common grade 3-4 AEs overall were neutropenic fever and pneumonia. Thus, this study found a safe sequential combination strategy for azacitidine and lenalidomide for patients with higher-risk MDS, $75 \mathrm{mg} / \mathrm{m}^{2}$ intravenous azacitidine once a day for 5 days followed by $25 \mathrm{mg}$ oral lenalidomide once a day for 5 days. Also, this study provided preliminary evidence of activity of this schedule in patients with MDS.

\section{Lenalidomide - Summary and Current Status for its Use in Myelodysplastic Syndromes}

Lenalidomide is a novel thalidomide analogue with enhanced immunomodulatory and anti-angiogenic action lacking most of the typical thalidomide-associated AEs. Oral lenalidomide is approved in the US for the treatment of patients with transfusion-dependent anaemia due to lower risk (i.e. IPSS low- or intermediate-1-risk) del(5q) MDS, with or without additional cytogenetic abnormalities. ${ }^{31}$ While in Europe, it is indicated for the treatment of patients with transfusiondependent anaemia due to lower-risk MDS associated with an isolated del(5q) cytogenetic abnormality when other therapeutic options are insufficient or inadequate. ${ }^{32}$

According to the US National Comprehensive Cancer Network (NCCN) guidelines, patients with lower-risk del(5q) MDS who have clinically significant cytopenias and symptomatic anaemia should be treated with lenalidomide, along with supportive care as an adjunct to the treatment (category $2 \mathrm{~A}$ recommendation). ${ }^{33}$ Lenalidomide is also an option for patients with lower-risk non-del(5q) MDS, who have clinically significant cytopenias and symptomatic anaemia, with either a serum EPO level of $>500 \mathrm{mU} / \mathrm{mL}$ and a poor probability of responding to immunosuppressive therapy, or a serum EPO level of $\leq 500 \mathrm{mU} / \mathrm{mL}$ and who have not responded to ESA (category 2A recommendation). ${ }^{33}$ Guidelines from the European Society for Medical Oncology (ESMO) indicate that patients with $5 q$-syndrome may be treated with lenalidomide. ${ }^{4}$

The clinical efficacy of lenalidomide in patients with lower-risk del(5q) MDS observed in the phase II MDS-003 trial was further confirmed in the phase III MDS-004 trial. Treatment with lenalidomide in this population led to RBC-TI for $\geq 26$ consecutive weeks and cytogenetic responses in a significantly higher proportion of patients than placebo. Erythroid responses are achieved rapidly and about $60-67 \%$ of responders remained transfusion free at a median follow-up of 1.55 years. Lenalidomide generally has a manageable tolerability profile. The most common AEs occurring in lenalidomide recipients are thrombocytopenia and neutropenia, most cases can be managed by dosage reductions. Thus, lenalidomide is a useful option for the treatment of patients with lower-risk del(5q) MDS, with or without additional cytogenetic abnormalities. Recently published results 
of MDS-005 also suggested its role also in lower-risk MDS without del $(5 q) .{ }^{27}$ Recent trials have focused on combining lenalidomide with other agents active for treatment of higher-risk MDS. ${ }^{29,30}$

There are currently no sufficient data elucidating long-term benefits of Ienalidomide therapy. Moreover, it remains unclear to decide when it can be discontinued. Further studies investigating the role of lenalidomide in lower-risk MDS without del(5q) and its role in higher-risk MDS in combination with other agents are warranted, which can give more clarity on the dosing schedule and efficacy of such combinations. Studies assessing the impact of long-term immunomodulation with lenalidomide and optimum duration of therapy in MDS patients would also be of interest.
1. Nimer SD, Myelodysplastic syndromes, Blood 2008;111:4841-51.

2. Jädersten $M$, Hellström-Lindberg $E$, Myelodysplastic syndromes: biology and treatment, $J$ Intern Med 2009;265:307-28.

3. Rollison DE, Howlader N, Smith MT, et al., Epidemiology of myelodysplastic syndromes and chronic myeloproliferative disorders in the United States, 2001-2004, using data from the NAACCR and SEER programs, Blood, 2008;112:45-52

4. FenauX P, Haase D, Sanz GF, et al., Myelodysplastic syndromes: ESMO Clinical Practice Guidelines for diagnosis, treatment and follow-up, Ann Oncol, 2014;25:57-69.

5. Greenberg P, Cox C, LeBeau MM, et al., International scoring system for evaluating prognosis in myelodysplastic syndromes, Blood, 1997;89:2079-88.

6. Greenberg PL, Tuechler $\mathrm{H}$, Schanz J, et al., Revised international prognostic scoring system for myelodysplastic syndromes, Blood, 2012;120:2454-65.

7. Haase $D$, Cytogenetic features in myelodysplastic syndromes, Ann Hematol, 2008;87:515-26.

8. Haase D, Germing U, Schanz J, et al., New insights into the prognostic impact of the karyotype in MDS and correlation with subtypes: evidence from a core dataset of 2124 patients, Blood, 2007;110:4385-95

9. Vardiman JW, Thiele J, Arber DA, et al., The 2008 revision of the World Health Organization (WHO) classification of myeloid neoplasms and acute leukemia: rationale and important changes, Blood, 2009;114:937-51.

10. Fenaux P, Kelaidi $C$, Treatment of the $5 q$ - Syndrome Hematology, 2006;2006:192-8

11. Mallo M, Cervera J, Schanz J, et al., Impact of adjunct cytogenetic abnormalities for prognostic stratification in patients with myelodysplastic syndrome and deletion $5 q$ Leukemia, 2011:25:110-20.

12. Kotla V, Goel S, Nischal S, et al., Mechanism of action of Kotla V, Goel S, Nischal S, et al., Mechanism of action of
lenalidomide in hematological malignancies, $J$ Hematol Oncol 2009;2:36.

13. Anderson $\mathrm{KC}$, Lenalidomide and thalidomide: mechanisms of action - similarities and differences, Semin Hematol, 2005;42(Suppl. 4):S3-8.

14. Galustian C, Meyer B, Labarthe M-C, et al., The anti-cancer agents lenalidomide and pomalidomide inhibit the proliferation and function of $\mathrm{T}$ regulatory cells, Cancer Immunol and function of T regulatory cells,

15. Heise C, Carter T, Schafer P, Chopra R, Pleiotropic mechanisms of action of lenalidomide efficacy in del(5q) myelodysplastic syndromes, Expert Rev Anticancer Ther, 2010;10:1663-72

16. Padron E, Komrokji R, List AF, Biology and treatment of the $5 q$ syndrome, Expert Rev Hematol, 2011;4:61-9.

17. Wobus M, Benath $\mathrm{G}$, Ferrer RA, et al., Impact of Ienalidomide on the functional properties of human mesenchymal stromal cells, Exp Hematol, 2012;40:867-76.

18. Hoefsloot $L H$, van Amelsvoort MP, Broeders $L C$, et al., Erythropoietin-induced activation of STAT5 is impaired in the myelodysplastic syndrome, Blood, 1997;89:1690-700.

19. Dredge $K$, Marriott JB, Macdonald $C D$, et al., Novel thalidomide analogues display anti-angiogenic activity independently of analogues display anti-angiogenic activity independently

20. List A, Kurtin S, Roe DJ, et al., Efficacy of Ienalidomide in myelodysplastic syndromes, N Eng/ J Med, 2005;352:549-57.

21. List A, Dewald G, Bennett J, et al., Lenalidomide in the myelodysplastic syndrome with chromosome $5 q$ deletion, N Engl J Med, 2006;355:1456-65.

22. Fenaux $P$, Giagounidis $A$, Selleslag $D$, et al., A randomized phase 3 study of lenalidomide versus placebo in RBC transfusion-dependent patients with Low-Intermediate1-risk myelodysplastic syndromes with del5q, Blood, 2011;118:3765-76.

23. Kuendgen A, Lauseker M, List AF, et al., Lenalidomide does not increase AML progression risk in RBC transfusiondependent patients with Low- or Intermediate-1-risk MDS with del(5q): a comparative analysis, Leukemia 2013;27:1072-9.

24. Fenaux P. Haase D, Sanz GF et al, Myelodysplastic syndromes: ESMO Clinical Practice Guidelines for diagnosis, treatment and follow-up, Ann Oncol, 2014;25(Suppl. 3):iii57-69.

25. Revicki DA, Brandenburg NA, Muus P, et al., Health-related quality of life outcomes of lenalidomide in transfusiondependent patients with Low- or Intermediate-1-risk myelodysplastic syndromes with a chromosome $5 q$ deletion: results from a randomized clinical trial, Leuk Res,
2013;37:259-65

26. Raza A, Reeves JA, Feldman EJ, et al., Phase 2 study of lenalidomide in transfusion-dependent, low-risk, and intermediate-1-risk myelodysplastic syndromes with karyotypes other than deletion 5q, Blood, 2008;111:86-93.

27. Santini V, Almeida A, Giagounidis A, et al., Efficacy and safety of lenalidomide (LEN) versus placebo (PBO) in RBC-transfusion dependent (TD) patients (pts) with IPSS low/intermediate (Int-1)-risk myelodysplastic syndromes (MDS) without Del(5q) and unresponsive or refractory to erythropoiesis-stimulating agents (ESAs): Results from a randomized phase 3 study (CC-5013-MDS-005), Blood, 2014;124:409-409.

28. Sekeres MA, Tiu RV, Komrokji R, et al., Phase 2 study of the lenalidomide and azacitidine combination in patients with higher-risk myelodysplastic syndromes, Blood 2012;120:4945-51.

29. Sekeres MA, Othus $M$, List $A F$, et al., A randomized phase II study of azacitidine combined with lenalidomide or with vorinostat vs. azacitidine monotherapy in higherrisk myelodysplastic syndromes (MDS) and chronic myelomonocytic leukemia (CMML): North America Intergroup Study SWOG S1117. Presented at American Society of Hematology (ASH) Annual Meeting, 6-9 December 2014; San Francisco, CA. Abstract LBA-5.

30. DiNardo $C D$, Daver $N$, Jabbour $E$, et al., Sequential azacitidine and lenalidomide in patients with high-risk myelodysplastic syndromes and acute myeloid leukaemia: a single-arm, phase 1/2 study, Lancet Haematol, 2015;2:e12-20.

31. NCCN Clinical Practice Guidelines in Oncology [Internet]. Available from: http://www.nccn.org/ (accessed 30 August 2015).

32. Celgene Corporation. REVLIMID [lenalidomide] capsules, for oral use: prescribing information. [Internet]. 2013. Available from: http://www.accessdata.fda.gov/drugsatfda_docs/ label/2013/021880s038lbl.pdf (accessed 30 August 2015).

33. European Medicines Agency. Revlimid (lenalidomide): summary of product characteristics. 2013 [Internet]. Available from: http://ec.europa.eu/health/documents/communityregister/2013/20130613126109/anx_126109_en.pdf (accessed 11 November 2015) 